\title{
Transumbilical single-incision laparoscopic hysterectomy for large uterus: feasibility of the technique
}

\author{
Juan Gilabert-Estelles • Jose Manuel Castello • \\ Juan Gilabert-Aguilar
}

Received: 24 August 2009 / Accepted: 4 November 2009 / Published online: 26 November 2009

(C) Springer-Verlag 2009

\begin{abstract}
Total hysterectomy has been shown to have more clinical benefits when performed with a laparoscopic approach in comparison to traditional open surgery. However, multiple puncture sites might increase trocarassociated complications, such as bleeding, hernias, and wound infection and the cosmetic results are not always optimal. The umbilicus, an embryonic natural orifice, is an anatomical structure that may be used to perform advanced gynecological procedures, further reducing the morbidity associated with classical laparoscopic surgery. Leiomyomas are one of the most common indications for hysterectomy in women not wishing to conceive. Uterine size may be a limiting factor for laparoscopic single-incision approach to hysterectomy due to difficulties encountered to achieve a proper operative field. Several practical maneuvers can be used to assure a safe dissection while managing voluminous uteri. Herein we present our preliminary experience of transumbilical single-incision laparoscopic hysterectomy with a multichannel port in a patient affected of myomatous uterus.
\end{abstract}

Keywords Single port · Hysterectomy · Myomas · SILS . LESS · e-NOTES

J. Gilabert-Estelles · J. M. Castello · J. Gilabert-Aguilar Unit of Gynecological Endoscopy, Hospital Casa de Salud, Valencia, Spain

J. Gilabert-Estelles $(\varangle)$

Department of Gynecology, La Fe University Hospital, Avda. Campanar 21,

46009 Valencia, Spain

e-mail: juangilaeste@yahoo.es

J. Gilabert-Aguilar

Department of Gynecology, Hospital Arnau de Vilanova,

Valencia, Spain

\section{Introduction}

Laparoscopic surgery is a well-established alternative to open surgery for many benign and malignant gynecological procedures. Since the first report of a total laparoscopic approach for hysterectomy 20 years ago [1], encouraging efforts have been dedicated by many surgeons in order to reduce the overall abdominal wall trauma by decreasing either the size of ports and the number of trocars.

Although laparoscopy is less invasive than laparotomy, it still requires several incisions, each at least $5-10 \mathrm{~mm}$ in length. Even if internal organ damage is largely associated with the placement of the umbilical trocar, each abdominal scar increases the potential morbidity risks, such us bleeding, pain, or hernia, and may worsen the cosmetic results [2].

In the last years, advances in laparoscopic surgery have led to a novel surgical approach, known as single-incision laparoscopic surgery (SILS) or laparo-endoscopic singlesite surgery (LESS). Recently, new terminology has been described for the access through an embryonic natural orifice, such as the umbilicus, introducing the term of embryonic natural orifice transumbilical endoscopic surgery (e-NOTES). Single-incision approach to gynecological diseases is not a new idea. In 1991, Pelosi et al. [3] performed the first reported hysterectomy with a single umbilical puncture. Despite these efforts, SILS did not spread rapidly among gynecologic surgeons mostly due to technical difficulties in the absence of laparoscopic instrumentations appropriately adapted to the restricted operative field. Recently, with the advent of technological improvements, Nickles et al. reported 13 patients with gynecological oncological disorders treated through SILS, four of which were performed robotically with the daVinci ${ }^{\circledR}$ Surgical System (Intuitive Surgical, Sunnyvale, CA, USA) [5]. In single-incision approach, uterine size is 
regarded as one limiting factor to perform advanced procedures, as the partial loss in the triangulation of the instruments may hamper dissection movements in a distorted operative field. Herein we describe potential benefits and limitations of single-incision total laparoscopic hysterectomy in patients affected of uterine myomas.

\section{Case report}

By the beginning of April 2009, we started performing single-incision laparoscopic procedures through a multiple instrument access port (SILS Port ${ }^{\mathrm{TM}}$, Covidien, MA, USA) in five porcine models. We developed the modifications of our technique for advanced procedures, including hysterectomy, partial cystectomy, pelvic lymphadenectomy, vessel ligation, and intracorporeal suturing.

This multichannel port consists of a blue flexible softfoam port, with access channels for three cannulas. It allows the passage of up to three laparoscopic instruments (three 5-mm cannulas or two 5-mm and one 12-mm cannula; Fig. 1). After various attempts we decided to use a $30^{\circ} 5 \mathrm{~mm}$ laparoscope (Karl Storz, Tuttlingen, Germany), an articulated Maryland forceps, and scissors (Roticulator, Covidien, MA, USA) in order to optimize the angle of the approach to the target tissue. The three cannulas needed to be adjusted at different levels within the flexible port in order to avoid clashing of the instruments. $\mathrm{CO}_{2}$ insufflation was performed through a different channel at the lateral part of the SILS port.

Ligasure Advance ${ }^{\mathrm{TM}}$ sealing forceps with the Force Triad $^{\mathrm{TM}}$ energy platform (Valleylab, CO, USA) was used in order to achieve hemostasis of big vessels (Fig. 2). This instrument has new useful features, such as the sharp little monopolar 'tooth' attached to one of the jaws and

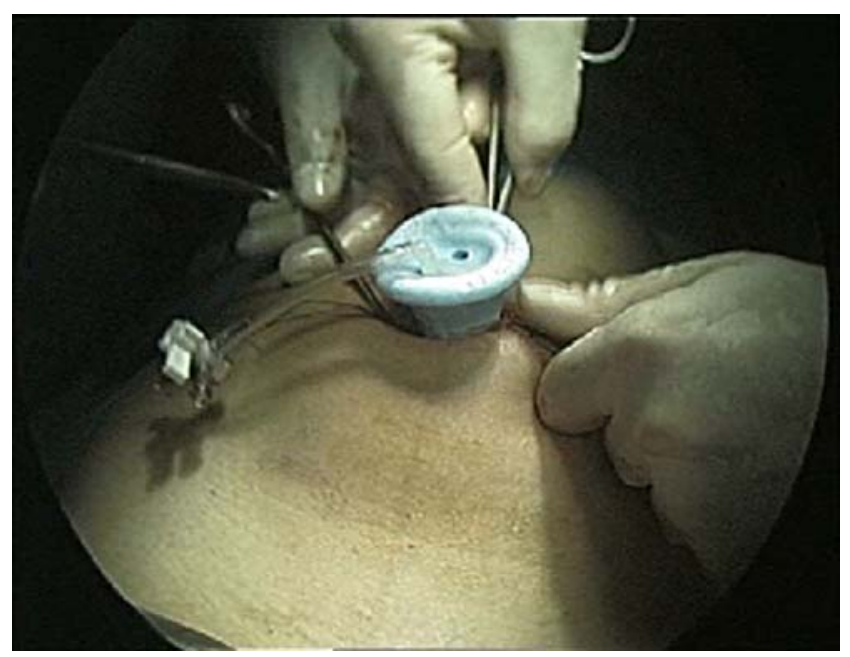

Fig. 1 External view of the multiple instrument access port the angled tip, which allows better visualization. The monopolar cutting capacity showed to be useful in the dissection of the spaces to allow a more safe approach to the uterine vascularization. Our successful experience in simulation surgery led us to transfer this technique to patients after obtaining the necessary institutional review board approval.

A 46-year-old Caucasian multigravid woman was referred to our endoscopy unit for severe anemia due to excessive bleeding during menses. The patient had a good physical status, a favorable body mass index (29), and a history of two births via vaginal delivery.

Gynecological examination revealed a 10th week voluminous uterus with a posterior myoma that partially obliterated the pouch of Douglas without any sign of pelvic organ prolapse. Vaginal ultrasound confirmed the presence of normal ovaries and the diagnosis of a $7-\mathrm{cm}$ posterior intramural myoma that distorted the endometrial cavity. Endometrial cytology and endometrial biopsy showed no alterations. An informed consent was obtained from the patient after discussing risks and benefits of a SILS approach. She was also counseled that additional incisions might be necessary as warranted during the surgical procedure. Our patient requested bilateral salpingooophorectomy (BSO) due to previous family history of gynecological malignancies.

Laparoscopy was performed under general endotracheal anesthesia with the patient in a dorsal lithotomy position. Standard bowel preparation was indicated and prophylactic intravenous antibiotic therapy (amoxicilin $2 \mathrm{~g}$ and $0.5 \mathrm{~g}$ of clavulanic acid) was given at the beginning of the operation. A Uterine Manipulator (Clermont-Ferrand, Karl Storz, Tuttlingen, Germany) was placed and a Foley catheter was inserted into the bladder. The umbilicus was pulled up with an atraumatic forceps and a $2 \mathrm{~cm}$ incision

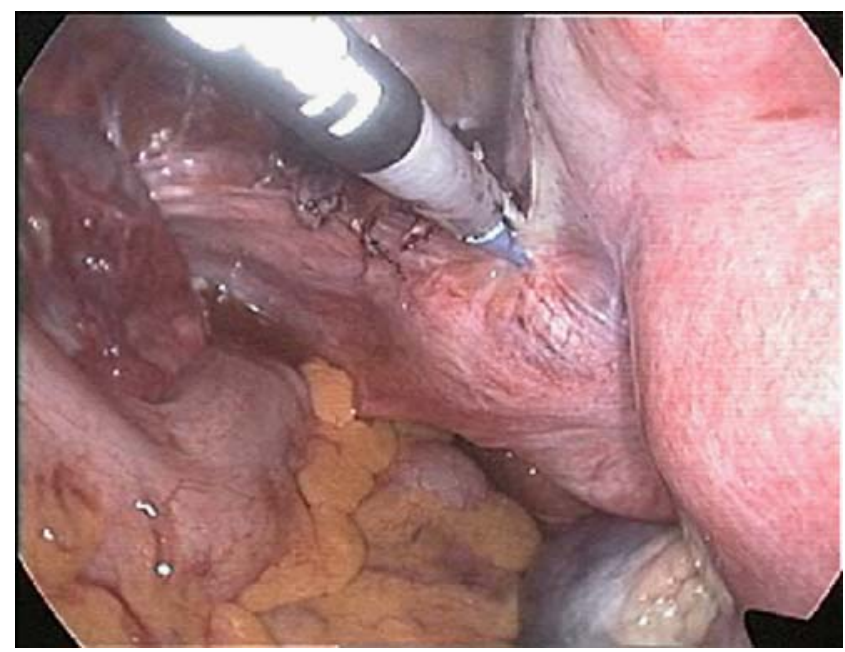

Fig. 2 Ligasure sealing forceps is used at the left uterine vessels 
was made without passing its outer folds. Using an open Hasson technique, the fascia was identified and polyglactin 0 sutures were placed in order to facilitate the insertion of the trocar and the identification of the fascial edges, just before the closure of the abdomen. The incision of the fascia needed to fit the trocar diameter in order to avoid pneumoperitoneum due to gas leak around the flexible port. Next, the flexible SILS port was covered with vaseline, then folded at its lower edge with a clamp and introduced into the abdominal cavity. The three cannulas were inserted and the $30^{\circ}$ optic confirmed the correct visualization of the intraperitoneal structures before the establishment of the pneumoperitoneum. A 5-mm Ligasure device was inserted through the most cranial cannula and both the roticulator articulated forceps and the scissors were used alternatively through the remaining free cannula. The patient was then placed in the usual $30^{\circ}$ Trendelenburg position and pelvic examination detected the presence of a myomatous uterus with a voluminous posterior myoma. The anteroposterior and lateral mobilization of the uterus with the manipulator showed to be enough to proceed with the hysterectomy laparoscopically.

Inspection of the pelvis permitted the identification of both ureters. Round ligament was retracted laterally with the articulated Maryland forceps, while the uterus was displaced medially with the uterine manipulator (Fig. 3). Ligasure allowed a correct sealing of the ligament and a monopolar cutting at the tip of the instrument facilitated the dissection of the anterior leaf of the broad ligament. Next, a window was performed at the posterior leaf of the broad ligament in order to caudally displace the ureter. The infundibulopelvic ligament was sealed and cut while the traction forceps moved the adnexa medially (Fig. 4). The articulated forceps was used to retract the bladder at the midline towards the abdominal wall while the scissor opened and dissected the

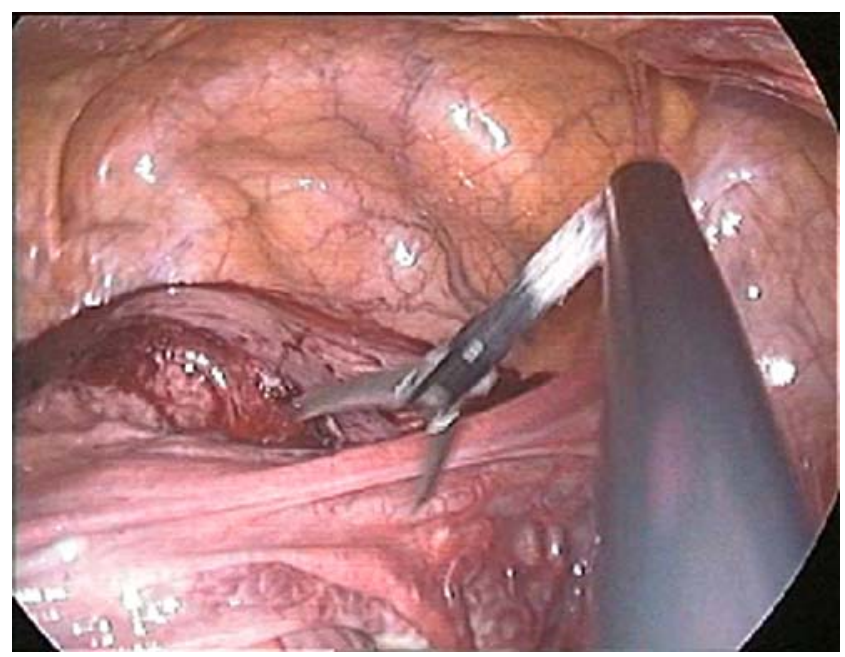

Fig. 3 Articulated Maryland forceps grasps the round ligament

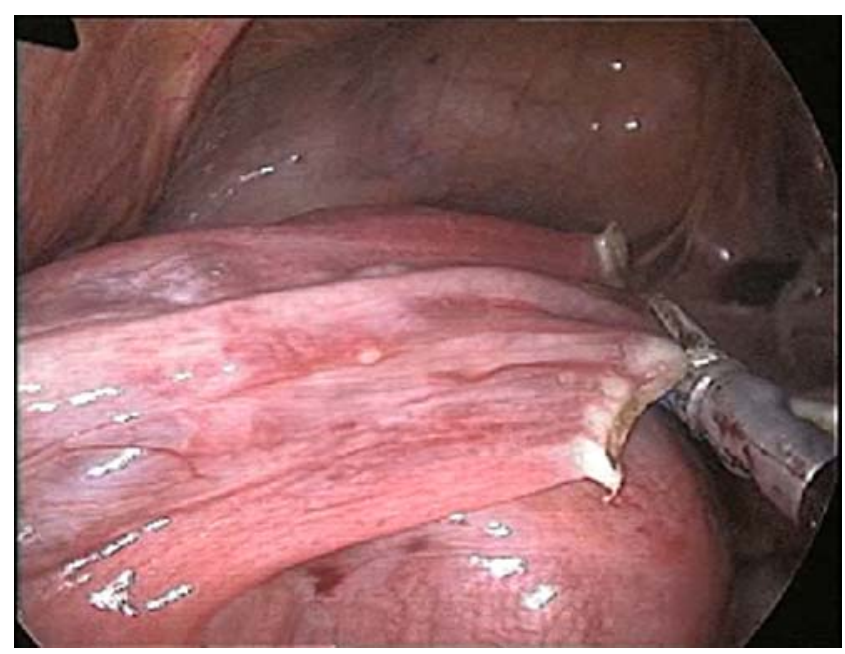

Fig. 4 Infundibulopelvic ligament is coagulated and sectioned while the adnexa is retracted medially

vesicovaginal space. This maneuver permitted a safe section of the bladder pillars. Uterine artery and vein were sealed using the Ligasure system, but difficulties were encountered in achieving a proper angle between the forceps and the vessels. Uterine lateralization with the manipulator facilitated this step.

The procedure was then repeated at the right side. The manipulator's vaginal valve was then inserted and a circumferential colpotomy was easily performed with the articulated scissors connected to the monopolar courant. The removal of the uterus was then performed vaginally. The cuff of the vagina was laparoscopically sutured with interrupted 0 -vicryl sutures managed with a standard 5-mm needle holder with the assistance of the Maryland articulated forceps. Intracorporeal knotting technique allowed the cuff to be sutured despite difficulties arising in relation to the reduced angle, the clashing of the instruments, and the considerable distance between the vagina and the umbilicus (Fig. 5). Fascia was closed by approximating the previously placed 0 -polyglactin sutures. The uterus weighted $480 \mathrm{~g}$.

The operating time was $125 \mathrm{~min}$ and the patient was discharged uneventfully $18 \mathrm{~h}$ after surgery, being the drop of hemoglobin levels of $0.8 \mathrm{~g} / \mathrm{dL}$. Pathological exam of the uterus confirmed the diagnosis of myomatous uterus. To date, follow-up has reached 3 months, and the patient remains asymptomatic.

\section{Discussion}

Gynecologists have put great efforts to further reduce the morbidity and improve the cosmetic results of laparoscopic surgery, including the reduction of the size and number of the ports. 


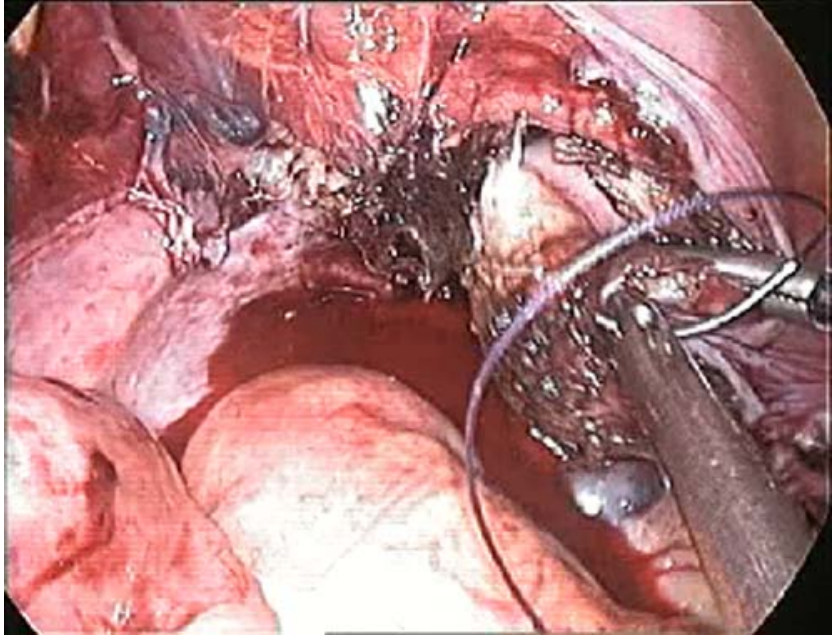

Fig. 5 Suturing of the vagina was performed with laparoscopic intracorporeal knots

Three decades ago, Wheeless and Thompson $[6,7]$ first published the technique and the results of a large series of laparoscopic tubal ligation with single-trocar laparoscopy. In 1991, Pelosi et al. performed the first complex gynecological procedure, a total hysterectomy with BSO, using the single-puncture technique [3]. One year thereafter the first supracervical hysterectomy was performed for benign uterine diseases in four patients, introducing the term of minilaparoscopy [8]. Unfortunately, this technique has not been successfully spread among gynecologists mainly due to technical difficulties and a lack of specific instrumentations allowing and safe approach for difficult cases.

New instrument development has prompted many surgeons to re-introduce the SILS for a variety of procedures. In 1998, a single-incision laparoscopic appendectomy [9] was described, in which the appendix was laparoscopically mobilized and the appendectomy was extracorporeally performed through a single umbilical incision. Soon, other basic and advanced procedures were reported, such as hysterectomy, nephrectomy, pyeloplasty, cholecystectomy, and right hemicolectomy [10-14].

The experience with single-port access in 24 patients treated by laparoscopic-assisted vaginal hysterectomy by using a home-made multiple channel port has been recently reported [10]. Interestingly, the uterine weight was equal to other series reported for classical laparoscopic hysterectomy and the success rate of $87.5 \%$ was acceptable and included the learning curve of the surgeon. Unfortunately no comments were underlined in relation to difficulties concerning the vaginal cuff closure through the single-incision approach. In the present report, some difficulties were encountered when suturing the vagina through the umbilicus. Firstly, the distance from the vagina to the umbilicus led to a non-ergonomic working position in which the needle holder has nearly all its length inside the abdomen, thereby reducing the precision of the movements at the tip. In addition, the lack of articulation of the needle holder and the reduced triangulation made intracoroporeal knotting more difficult. Pushing the vagina with a vaginal probe and using the articulated forceps set at maximal angle allowed us to have a better exposure to achieve the laparoscopical suture of the vagina.

Even though SILS avoids multiple incisions and uses an embryonic natural orifice such as the umbilicus, some criticisms have been remarked regarding the lack of data supporting a real benefit for patients when compared to standard open or multiport laparoscopic techniques. In addition, the increase of costs related to new instrumentation may be a limiting factor of this new approach. There are few reports in the literature concerning the possible advantages of this single-incision access.

In the series reported by Lee et al., the overall outcome of SILS for hysterectomy was comparable to conventional methods [10], therefore demonstrating the feasibility of the technique. Other authors have confirmed the feasibility of this technique [15]. Larger studies though should be designed in order to establish the exact clinical benefits of this approach.

Raman et al. reported the perioperative outcomes of SILS versus conventional laparoscopic nephrectomy. Both groups were comparable in terms of operative time, decrease of hemoglobin, analgesic use, and length of stay, and the authors concluded that this possible lack of difference of the techniques might be related to the extended expertise of the surgeons after multiple SILS nephrectomy cases in a porcine model [11]. Prior experience in animal laboratory is of utmost importance when moving from classical laparoscopic approach to SILS. Specific technical conditions of single-port access necessitate a new learning curve even for most trained surgeons. Our preliminary experience in five porcine models allowed us to better chose the instrumentation, to assay different combinations for its placement through the cannulas, and to learn the proper management of bleeding and the suturing technique. Although the relatively thin porcine abdominal wall makes the introduction of the SILS port easier than in humans, the distribution and proportion of the viscera in this model is so similar to those in humans, that it could actually represent a valuable tool to train difficulties that may be encountered in real surgery.

On one hand, a number of advantages of SILS have been proposed including cosmetic results, with practically no incision seen by the patient, less incisional pain, and the ability to convert to standard multiport laparoscopic surgery if needed. On the other, the restricted freedom of movement, the reduced number of cannulas that can be 
used, and the proximity of the instruments to each other during the operation probably account for the most relevant disadvantages of SILS. These conditions might lead to a lack of proper triangulation; a need for special instrumentation, instrument crowding, or clashing; and frequent antiergonomic position of the instruments. All of these aspects, that increase the complexity and technical challenges of the operation, could be significantly reduced after a learning curve period has been fulfilled in a porcine model.

Instrument triangulation is a necessary condition in laparoscopic surgery as it allows an appropriate tissue retraction, which is the basis for an accurate dissection along the anatomical surgical planes. Placing parallel instruments through a single site, makes triangulation more difficult as movements of the hands usually lead to crossing and clashing of the instruments. Several tips may be used to reduce this possibility. Firstly, using at least one instrument with articulating capacity is often enough to obtain some degree of triangulation. The management of this type of instrument is usually difficult due to the requirement of counterintuitive movements. Therefore, non-trained surgeons can use this forceps at maximum angulation as a static traction device that facilitates the use of a nonarticulating sealing or cutting instrument. Secondly, additional assistance, such as a concomitant uterine manipulator can be of invaluable help, provide the right angle during dissection and allow a better exposure of the field. In addition, transparietal sutures using a straight needle can be placed with the assistance of the instruments used through the SILS trocar. This maneuver might be useful to retract those structures which are usually displaced, such as the ovaries and the bowels. It is advisable not to tie the suture in order to retain the possibility to maintain a variable traction potential as dissection proceeds.

External crowding and clashing of the handle of instruments is one of the most frustrating aspects of the learning curve of SILS. It is important to set the cannulas at different levels through the multichannel trocar in order to prevent the external handle of the instruments from clashing. Furthermore, the rotation skills of the instruments and the continuous modification of the angle of the camera during the surgery are also very useful to avoid this problem.

Imaging represents another important issue when working through a single port, since depth perception and angles are generally distorted. We used a $30^{\circ} 5 \mathrm{~mm}$ optic in order to maintain the possibility of using $10-\mathrm{mm}$ instruments and have different viewing angles depending on the placement of the instruments. During the procedures, the instruments frequently cross over the field of visualization of the laparoscope. Therefore, the assistant must constantly reorient the camera in order to anticipate clashing and obtain the better vision of the field.
The myomatous uterus of the herein presented case arouses serious concerns about the possibility of a subsequent restriction in the approachability. Umbilicus in obese patients is frequently situated in a more caudal position. Therefore, the umbilical access may not be appropriate in some obese patients with very large uterus. Meanwhile, even if our patient had a voluminous posterior intramural myoma, the working distance was adequate. Uterine anteversion with the manipulator showed to be very useful to perform the posterior dissection and also to approach the uterine vessels. It should be noted that nonarticulated instruments tend to proceed more laterally as dissection advance and this behavior might be dangerous while coagulating at the parametrium. An angled sealing instrument permitted us to correct this trend and achieve a proper and safe sealing of the uterine vessels.

There is growing experience supporting the use of robotics applied to gynecological surgery in the last years. Even though robotics usually needs multiple $8 \mathrm{~mm}$ ports, Escobar et al. [4] have recently reported their initial clinical experience and technique in gynecology with robotic-assisted single-port surgery. The ability of the robotic arms to broaden the surgeon's mobility and the triangulation potential has deeply improved ergonomics, thereby substantially reducing the difficulties encountered with classical SILS. According to the author's experience, even though the instruments are placed in parallel, the wide range of movements of the robotic system enables an accurate tissue dissection. Probably, when these technologies become widely available, SILS procedures will also advance even further and limitations of the technique will decrease.

In conclusion, single-incision total laparoscopic hysterectomy is a promising approach for benign conditions that may decrease trocar-site-related complications and improve cosmetics and patient recovery, maintaining the ability to convert the procedure to a standard laparoscopy if special difficulties arise. The animal model represents a unique opportunity to acquire expertise in the technique, practice the herein presented suggestions to improve the procedure, or even find other modifications with the same goal. The era of this new minimally invasive operative approach is maybe just about to come.

Conflict of interest There is no actual or potential conflict of interest in relation to this article.

\section{References}

1. Reich H, DeCaprio J, McGlynn F (1989) Laparoscopic hysterectomy. J Gynecol Surg 5:213-217 
2. Canes D, Desai MM, Aron M et al (2008) Transumbilical singleport surgery: evolution and current status. Eur Urol 54:1020-1030

3. Pelosi MA, Pelosi MA 3rd (1991) Laparoscopic hysterectomy with bilateral salpingo-oophorectomy using a single umbilical puncture. N J Med 88:721-726

4. Escobar PF, Nickles Fader A, Paraiso MF, Kaouk JH, Falcone T (2009) Robotic-assisted laparoendoscopic single-site surgery in gynecology: initial report and technique. Journal of Minimally Invasive Gynecology 16:589-591

5. Nickles Fader A, Escobar PF (2009) Laparoendoscopic single-site surgery (LESS) in gynecologic oncology: technique and initial report. Gynecol Oncol 114:157-161

6. Wheeless CR (1969) A rapid, inexpensive and effective method of surgical sterilization by laparoscopy. J Reprod Med 3:65-69

7. Wheeless CR Jr, Thompson BH (1973) Laparoscopic sterilization: review of 3600 cases. Obstet Gynecol 42:751-758

8. Pelosi MA, Pelosi MA 3rd (1992) Laparoscopic supracervical hysterectomy using a single-umbilical puncture (minilaparoscopy). J Reprod Med 37:777-784
9. Esposito C (1998) One-trocar appendectomy in pediatric surgery. Surg Endosc 12:177-178

10. Lee Y-Y, Kim T-J, Kim CJ et al (2009) Single-port access laparoscopic-assisted vaginal hysterectomy: a novel method with a wound retractor and a glove. Journal of Minimally Invasive Gynecology 16:450-453

11. Raman JD, Bensalah K, Bagrodia A, Stern JM, Cadeddu JA (2007) Laboratory and clinical development of single keyhole umbilical nephrectomy. Urology 70:1039-1042

12. Desai MM, Rao PP, Aron M et al (2008) Scarless single-port transumbilical nephrectomy and pyeloplasty: first clinical report. BJU 101:83-88

13. Bucher P, Pugin F, Buchs N, Ostermann S, Charara F, Morel P (2009) Single-port access laparoscopic cholecystectomy. World J Surg 33:1015-1019

14. Bucher P, Pugin F, Morel P (2008) Single-port access laparoscopic right hemicolectomy. Int J Colorectal Dis 23:1013-1016

15. O'Hanlan KA (2009) First single-incision type 7 total laparoscopic hysterectomy, adnexectomy, and appendectomy. Gynecological Surgery. doi:10.1007/s10397-009-0509-z 